Wettasinghe, A. H., Dissanayake, D. W. N., Allet, L., Katulanda, P., \& Lord, S. R. (2020). Falls in older people with diabetes: Identification of simple screening measures and explanatory risk factors. Primary Care Diabetes.

DOI : $10.1016 / \mathrm{j} . \mathrm{pcd} .2020 .05 .006$

\title{
Falls in older people with diabetes: Identification of simple screening measures and explanatory risk factors
}

\begin{abstract}
Aims: To identify risk factors for falls in older people with diabetes mellitus (DM) and to develop a low-cost fall risk screening tool.
\end{abstract}

Methods: Older adults with DM (n=103; age=61.6 \pm 6.0 years) were recruited from diabetic clinics. Demographic, DM specific factors, lower limb strength and sensation, cognition, fear of falling, hand reaction time, balance, mobility and gait parameters were assessed using validated methods. Falls were prospectively recorded over six months.

Results: Past falls and female gender were identified as significant predictors of falls: history of falls and female gender increased fall rates by $4.62(95 \% \mathrm{CI}=2.31$ to 9.27$)$ and $2.40(95 \% \mathrm{CI}=$ 1.04 to 5.54) respectively. Fall rates were significantly associated with Diabetic Neuropathy scores, HbA1c level, contrast sensitivity, quadriceps strength, postural sway, tandem balance, stride length and Timed Up and Go Test times. A multi-variable fall risk tool derived using five measures, revealed that absolute risk for multiple falls increased from $0 \%$ in participants with zero or one factor to $83 \%$ in participants with all five risk factors.

Conclusions: Simple screening items for fall risk in people with DM were identified, with parsimonious explanatory risk factors. These findings help guide tailored interventions for preventing falls in DM.

Key words- accidental falls; aged; balance; diabetes; diabetic neuropathy; muscle strength

\author{
Abbreviations \\ BMI- body mass index \\ DM- Diabetes mellitus \\ Icon-FES- Iconographical Falls Efficacy Scale \\ IRR-incidence rate ratio \\ MOCA- Montreal Cognitive Assessment Test \\ MET- Melbourne Edge Test \\ TMT- Trail Making Test \\ TUGcog- cognitive dual task TUG \\ TUG- Timed Up and Go Test
}




\section{INTRODUCTION}

Diabetes mellitus is now a worldwide pandemic, with two thirds of the global diabetes population living in the developing countries [1]. In Sri Lanka the diabetes mellitus prevalence has increased dramatically over the past 15 years in both urban and rural districts with major impacts on sufferers and the health care system [1]. According to the International Diabetes Federation, the prevalence of diabetes mellitus in adults in Sri Lanka reached 8.6\% in 2017 [2].

Falls represents one of the major health care issues for people with diabetes mellitus. Approximately $39 \%$ of people with diabetes mellitus fall one or more times within a year [3]. Falls can result in injuries ranging from lacerations, bruises and abrasions, through to dislocations, sprains, fractures and traumatic brain injury [4]. Falls can also lead to decreased functioning in daily life, social isolation, fear of falling, loss of independent living and reduced quality of life [5-9].

Previous studies have found that in addition to poor diabetes control [10], diabetic complications [10], advanced disease status [11], sensory loss [12], muscle weakness [13], increased postural sway [14], gait and mobility impairments [15, 16], foot and body pain [17], pharmacological complications [17] and fear of falling [18] increase fall risk in people with diabetes mellitus. In addition, although not providing insight into why future falls occur, "past falls" has been shown to be a useful screening variable for fall risk [19]. However, previous studies have used only a limited range of tests to assess potential risk factors for falls, and some have used expensive high-tech equipment that is unavailable in most clinical settings throughout the world. Furthermore, a simple low-cost fall risk screen has yet to be developed for people with diabetes mellitus.

In this study, we assessed specific impairments across diabetes mellitus -specific, cognitive, sensorimotor, balance and functional mobility domains to identify risk factors for falls in a community-living sample of people with diabetes mellitus. We aimed to devise (1) a simple screening tool and (2) an explanatory assessment that elucidates the pathophysiology of falls in people with diabetes mellitus to guide intervention strategies for fall prevention.

\section{MATERIAL AND METHODS}

\subsection{Study design}

This study employed a prospective cohort design with six months falls follow-up. All participants underwent base-line assessments including assessments for neuropathy. Ethics approval was obtained from the Ethics Review Committee of the Faculty of Medicine, University of Colombo, Sri Lanka and from the National Hospital Colombo, Sri Lanka. Informed verbal and written consent were obtained from all the participants prior to study participation.

\subsection{Participants}

Participants were recruited from March 2017 to May 2018 from endocrinology clinics at the National Hospital, Colombo, Sri Lanka. Inclusion criteria included having diabetes more than 5 years, aged 50-70 years, living in the community, able to understand instructions necessary for 
the assessments and able to ambulate household distances without an assistive device.

Participants with significant central nervous system dysfunctions, musculoskeletal deformity or lower limb pathologies that affect balance were excluded. The sample size was chosen to allow for up to three predictors in multivariate models with at least 10 outcome cases per predictor variable [20].

\subsection{Baseline assessments}

\subsubsection{Demographics, health status, fear of falling}

Participants completed a questionnaire pertaining to demographics, falls in the previous year, diabetes-related factors and medication use. Peripheral neuropathy was defined as the presence of three of the following a) a validated Diabetic Neuropathy Symptom (DNS) score $\geq 1[21]$, b) an Diabetic Neuropathy Examination (DNE) score $\geq 4$ [21], c) a nerve conduction time (tibial nerve velocity) $<40 \mathrm{~m} / \mathrm{s}$ [21] and d) a vibration perception threshold $>25 \mathrm{~V}$ (21) (see details below). In addition, we measured HbA1c to assess glycemic control. Level of concern about falls was assessed with the Iconographical Falls Efficacy Scale (Icon-FES) [22], which uses pictures and matching short phrases to assess level of concern about falls for a range of everyday activities.

\subsubsection{Cognition and neuropsychological functioning}

The Montreal Cognitive Assessment Test (MOCA) [23] was used to assess general cognition. Cognitive motor speed and task switching ability, aspects of executive function, were measured using the Trail Making Test (TMT) [24]. Part A requires participants to draw lines connecting numbers (e.g., 1-2-3), and Part B requires participants to draw lines connecting alternating letters and numbers (e.g., 1-A-2-B). The difference between the two parts was calculated to remove the speed element from the test evaluation. Simple reaction time in milliseconds was assessed using a light as a stimulus and a finger-depression of a switch as the response.

\subsubsection{Lower limb sensation}

Tibial nerve conduction velocity was assessed with Natus Xltek nerve conduction device (Koll Center Parkway Suite, Pleasanton, CA, USA). Vibration perception thresholds of the big toe were measured using a Biothesiometer (Bio Medical Instrument co, Ohio, USA). Tactile sensitivity was assessed with a Semmes-Weinstein pressure aesthesiometer comprising 20 nylon filaments of equal length with varying diameter. The filaments were applied to the center of the lateral malleolus, and pressure measurements were expressed as logarithms of the bending force in milligrams. Lower limb proprioception was measured using a lower limb matching task with participants sitting and eyes closed [25]. Errors in matching the great toes were recorded using a protractor inscribed on a vertical clear acrylic sheet $(60 \times 60 \times 1 \mathrm{~cm})$ placed between the legs.

\subsection{4. $\underline{\text { Vision }}$}

High and low contrast visual acuity were tested with a LogMAR letter chart [26] positioned $3 \mathrm{~m}$ in front of participants and measured under binocular conditions with participants wearing their distance correction glasses if applicable. Visual contrast sensitivity was assessed using the 
Melbourne Edge Test (MET) [25], which presents 20 circular patches containing edges with reducing contrast. Correct identification of the orientation of the edge on the patches provides a measure of contrast sensitivity in decibel units, where $1 \mathrm{~dB}=10 \log 10$ contrast.

\subsubsection{Muscle strength, balance, gait and mobility}

Maximal isometric quadriceps strength was measured in both legs while participants were seated on a high chair (so that feet did not touch the floor) with the hips and knees flexed to $90^{\circ}$ [25]. A strain gauge was fixed horizontally with straps on the lower shin, $10 \mathrm{~cm}$ above the ankle, after which the participant was given three attempts with the dominant leg to push against the strap as forcefully as possible. In addition, the five time sit to stand test was administered [27]. Participants were given a practice trial and then the second trial was taken as the test result.

Postural sway was assessed using a sway meter that measured displacements of the body at the waist [28]. Testing was performed with participants standing on the floor and on a foam rubber mat $(40 \times 40 \times 15 \mathrm{~cm}$ thick) with eyes open and closed. Sway path (number of mm squares traversed by the sway meter pen) for each $30 \mathrm{~s}$ test was recorded. Two measures of leaning balance were administered - the maximal balance range test [29] which measure maximal foreaft maximal lean and the coordinated stability [29] which assesses participants' ability to adjust body position in a steady and coordinated way while placing them at or near the limits of their base of support . Standing balance was also assessed by time how long participants could stand on one leg [30] and feet in different positions [28, 31] (i.e. tandem and near tandem standing for 30 seconds).

Gait was assessed using the Gait-Up gait analysis system (Physilog5®, GaitUp; Lausanne, Switzerland) [32] that contains wearable sensors attached to the participant's left and right shoes or foot wear which they usually wear. Participants walked at their usual pace for $25 \mathrm{~m}$ along a corridor at the assessment site. During the analysis two steps were discarded from initiation and termination phases. Gait velocity, gait variability (coefficient of variation of cycle duration in percent), double support time, stance time, swing time, swing width, stride length and cadence were recorded.

Functional mobility was assessed with the Timed Up and Go Test [33] with (TUGcog) and without (TUG) a secondary cognitive task. Participants were asked to rise from a chair, walk forward three meters fast as they can, turn 180 degrees, walk back to the chair and sit down. The instructions were given as "standup, walk as quickly and safely as possible to the marked line, turn through 180 degrees, walk back to the chair, and sit down again". In TUGcog participants were asked to walk, while counting backward in threes from a randomly chosen start number between 60 and 100 .

\subsection{Falls}

Falls were defined as unexpected events which resulted in the participant unintentionally coming to the ground, floor or other lower level [34]. Participants were given 6 calendars at the baseline assessment and asked to record falls on the calendars each month and return them in pre-paid envelopes to the research centre. Participants who did not return calendars were telephoned by a research assistant to obtain the information. 


\subsection{Statistical analysis}

Initially, incidence rate ratios (IRRs) were calculated using negative binomial regression models assessing the associations between the individual demographic, health, and physical function assessment and falls. These models estimate the number of occurrences of an event when the event (such as falls) has Poisson variation with over-dispersion. Two subsequent multivariate regression models were then conducted: the first sought to identify the best set of independent and significant risk marker variables for use as a fall risk screen; the second sought to identify the best set of independent and significant explanatory risk factor variables for use as a fall risk assessment for guiding fall prevention strategies. Finally, one variable from five explanatory domains (lower-limb sensation / neuropathy, vision, lower limb strength, balance and gait / mobility found to be significantly associated with falls was selected for inclusion in a fall risk assessment tool. These variables were dichotomized, summed and contrasted against the occurrence of multiple falls in the follow-up period. Analyses were conducted using SPSS and STATA statistical software.

\section{RESULTS}

Characteristics of the 103 participants are described in Table 1. Falls data for the complete 6month follow-up period were available for all participants; 66 participants $(64.1 \%)$ had no fall, $11(10.7 \%)$ fell one time only and 26 participants $(25.2 \%)$ fell on two or more occasions during follow-up. 12 participants (32.4\%) suffered one or more fall-related injuries.

Demographic, medication, diabetes mellitus specific measures for the non-faller, single faller and multiple faller groups are shown in Table 1. Of these measures, female gender, a history of falls and greater fear of falling were identified as predictors of falls. Of the diabetes mellitus-specific factors, Diabetic Neuropathy Symptom score and Diabetic Neuropathy Examination score, $\mathrm{HbA1c}$ levels, insulin dependence and diagnosed neuropathy were significantly associated with falling. In contrast, increased age, reduced lower limb sensation (as measured by four validated tests), increased weight, higher BMI and greater medication use were not significantly associated with falls.

Table 2 presents the findings for the cognitive, vision, strength, gait and mobility measures for the non-faller, single faller and multiple faller groups. Several measures were significantly associated with falls: poor contrast vision, reduced knee extension strength, increased postural sway, reduced ability to tandem stand, slow walking speed, short stride length and slow TUG times (with and without a cognitive task). Notably, no cognitive measures were associated with falls.

The binomial regression multivariate model for the identification of screening measures revealed past falls and female gender as significant and independent predictors of falls (likelihood ratio Chi square $=48.91, \mathrm{p}<0.001)$. A history of one or more falls in the past year and female gender increased fall rates by factors of $4.62(95 \% \mathrm{CI}=2.31$ to 9.27$)$ and $2.40(95 \% \mathrm{CI}=1.04$ to 5.54$)$ respectively. The multivariate negative binomial regression model for the identification of explanatory measures comprised two significant and independent variables influencing falls: quadriceps strength and tandem balance ability (likelihood ratio Chi square $=12.43, \mathrm{p}=0.002$ ) . 
For each $1 \mathrm{~kg}$ increase in quadriceps strength fall rates decreased by $6 \%(\mathrm{IRR}=0.9495 \% \mathrm{CI}=$ 0.89 to 0.99 ) and the inability to undertake the tandem balance increased fall rates by a factor of $2.49(95 \% \mathrm{CI}=1.07$ to 5.77$)$.

The variables selected from the five explanatory domains for inclusion in a fall risk assessment tool comprised diagnosed neuropathy, poor contrast vision, reduced knee extension strength, poor balance and slow walking speed. Figure 1 shows the proportion of participants who suffered multiple falls in the follow-up period with respect the number of these risk factors present. Absolute risk for multiple falls ranged from $0 \%$ in those with zero or one risk factors to $83 \%$ in those with all five risk factors (Figure 1).

\section{DISCUSSION}

The main aims of this study were to devise a simple fall risk screen and a complementary assessment that elucidates the pathophysiology of falls in people with diabetes mellitus to guide intervention strategies for fall prevention. We found fall rates were significantly associated with previous falls, female gender, Diabetic Neuropathy Symptom and Diabetic Neuropathy Examination score, HbA1c levels, diagnosed neuropathy, poor contrast sensitivity, reduced quadriceps strength, increased postural sway, smaller stride length and poorer tandem balance, UST, and TUG test performances. Five measures: diagnosed neuropathy, poor contrast vision, reduced knee extension strength, poor balance and slow walking speed were included in a multivariable fall risk tool that revealed absolute risk for multiple falls over a 6-month follow-up period increased from $0 \%$ in participants with zero or one risk factor to $83 \%$ in participants with all five risk factors.

Past falls and female gender were the two strongest screening measures for falls. These observations are consistent with previous research demonstrating that past falls and female gender are risk factors for falls in people with diabetes mellitus $[19,35,36]$ and the general population of older people [37-39]. Yau et al. also reported female gender and falls in the past 12 months were risk factors for fall-injury related hospitalizations in people with diabetes mellitus [35]. The recording of past falls and female gender therefore merit inclusion in fall risk screens for identifying those in need of further assessment and subsequent fall prevention interventions. Such a screen could also contain the two independent explanatory measures (tandem balance ability and quadriceps strength) to comprise a simple four-item screen.

Several of the explanatory risk factors identified in this study have also been identified as fall risk factors in people with diabetes mellitus in previous studies. These include diabetic neuropathy [11], HbA1c levels [40] and insulin dependency [41], poor contrast vision [10], reduced lower limb strength [13], poor balance [42], slow gait speed [42] and impaired mobility [43]. Of these measures, the diagnoses and management of diabetic neuropathy appears crucial as it affects $50 \%$ of older people with diabetes mellitus and this condition influences almost all of the other identified fall risk factors. Lack of control of diabetes mellitus and insulin dependency may also increases fall risk as a result of hypoglycemia and subsequent clumsiness, confusion, loss of consciousness and seizures.

In a recent review, Hewston et al. [12] concluded cognitive decline is likely related to falls in people with diabetes mellitus, but that cognitive impairment has not yet been rigorously assessed 
as a fall risk factor in this population. In a local study, cognitive decline in elderly assessed with the MMSE and MOCA was suggested to be associated with increased risk of falls [44]. Interestingly, we did not find significant associations between falls and reduced general cognition as assessed with the MOCA or reduced executive functioning as assessed with the Trail Making tests in this study. The IRR for the dual task TUG time and falls was also smaller than that for the standard TUG test. It thus appears that the above tests either do not detect subtle cognitive impairments or that diabetes mellitus does not produce cognitive deficits in the diabetes mellitus population included here (mean age $\pm \mathrm{SD}=61.6 \pm 6.0$ years)

The findings of this study could be utilized for patient care as many of the fall risk factors identified are amenable to intervention. In particular, the five measures (diagnosed neuropathy, poor contrast vision, reduced knee extension strength, poor balance and slow walking speed) warrant inclusion in a fall-risk assessment tool. These assessments comprise either measures ascertained as part of routine care (diagnosed neuropathy) or simple low-tech tests with scope for widespread use in clinical settings. The findings that impaired strength, balance, gait and mobility were significant fall risk factors suggests that exercise would play a key role in addressing fall risk. This could be complemented with targeted interventions aimed at maximizing vision and diabetic control through education, medication prescription and lifestyle modification.

Strengths of this study include the broad range of putative risk factors, the prospective falls surveillance and the recruitment of a sample drawn from a community setting. These methodological procedures appear to have resulted in a representative sample of people with DM as the incidence of falls (i.e. 25\% falling in 6 months) was the same as that reported in a recent systematic review involving 1,692 people with DM [45]. However, we acknowledge certain study limitations. First, the inclusion of additional factors such as routine physical activity levels, and the presence of depression and pain may have assisted further in understanding why people with diabetes mellitus suffer falls. Second, our sample is relatively small for a prospective fall risk study, which likely limited multi-variable modelling. The study findings therefore require confirmation in external samples.

\section{CONCLUSIONS}

In conclusion, this study identified simple screening items for fall risk in people with diabetes mellitus, as well as a range of parsimonious explanatory risk factors across neuropathy, vision, strength, balance and mobility domains. These findings may assist in tailoring interventions for preventing falls in in people with diabetes mellitus.

\section{Acknowledgements}

The authors thank Director, National Hospital Colombo and staff at the participating diabetes clinics for their help in participant recruitment and data collection for the study. The authors also thank all the study participants for their immense contribution to the study. 


\section{Funding}

This work was supported by the Postgraduate research Scholarship (AP/3/2/2016/PG/05), University of Colombo, Sri Lanka. The study sponsor was not involved in the design of the study, the collection, analysis, and interpretation of data, writing the report or the decision to submit the report for publication.

\section{Declarations of interest}

The authors declare that there is no declarations of interest associated with this manuscript.

\section{Contribution statement}

All authors contributed equally in conceptualization of the study. Data collection was conducted by AHW under direct guidance and supervision of PK, SRL and DWND. AHW was trained for the methods of the study by SRL, PK and DWND. AHW prepared the manuscript under the guidance of SRL. SRL and DWND edited the manuscript. LA and PK contributed to the manuscript by editing and reviewing the manuscript. Final manuscript was reviewed and approved by all the authors for submission. 


\section{References}

1. Katulanda P, Sheriff MHR, R Matthews D. The diabetes epidemic in Sri Lanka - a growing problem. Ceylon Med J. 51, (2006), 26-34.

2. International Diabetes Federation (2019) South-East Asia, Sri Lanka [cited]. Available from https://www.idf.org/our-network/regions-members/south-east-asia/members/98-srilanka.html; Accessed 28 May 2019

3. Tilling LM, Darawil K, Britton M. Falls as a complication of diabetes mellitus in older people. J Diabetes Complications. 20, (2006), 158-162.

4. Schwenk M, Lauenroth A, Stock C, et al. Definitions and methods of measuring and reporting on injurious falls in randomised controlled fall prevention trials: a systematic review. BMC Med Res Methodol PMID. (2012);22510239. eng.

5. Sekaran NK, Choi H, Hayward RA, Langa KM. Fall-associated difficulty with activities of daily living in functionally independent individuals aged 65 to 69 in the United States: a cohort study. J Am Geriatr Soc. 61, (2013), 96-100.

6. Hajek A, König H-H. The association of falls with loneliness and social exclusion: evidence from the DEAS German Ageing Survey. BMC Geriatr. 17, (2017), 204.

7. Friedman SM, Munoz B, West SK, Rubin GS, Fried LP. Falls and fear of falling: which comes first? A longitudinal prediction model suggests strategies for primary and secondary prevention. J Am Geriatr Soc. 50, (2002), 1329-1335.

8. Downton JH, Andrews K. Prevalence, characteristics and factors associated with falls among the elderly living at home. Aging (Milano). 3, (1991), 219-228.

9. Hawkins K, Musich S, Ozminkowski RJ, et al. The burden of falling on the quality of life of adults with Medicare supplement insurance. J Gerontol Nurs. 37, (2011), 36-47.

10. Schwartz AV, Vittinghoff E, Sellmeyer DE, et al. Diabetes-related complications, glycemic control, and falls in older adults. Diabetes Care. 31, (2008), 391-197.

11. Richardson JK, Hurvitz EA. Peripheral neuropathy: a true risk factor for falls. J Gerontol A Biol Sci Med Sci. 50, (1995), M211-5.

12. Hewston P, Deshpande N. Falls and Balance Impairments in Older Adults with Type 2 Diabetes: Thinking Beyond Diabetic Peripheral Neuropathy. Can J Diabetes. 40, (2016), 6-9.

13. Macgilchrist C, Paul L, Ellis BM, et al. Lower-limb risk factors for falls in people with diabetes mellitus. Diabet Med. 27, (2010), 162-170.

14. Dixit S, Maiya A, Shasthry BA, et al. Postural sway in diabetic peripheral neuropathy among Indian elderly. Indian J Med Res. 142, (2015), 713-720.

15. Mustapa A, Justine M, Mohd Mustafah N, et al. Postural Control and Gait Performance in the Diabetic Peripheral Neuropathy: A Systematic Review. Biomed Res Int. 14, (2016)

16. Alvarenga PP, Pereira DS, Anjos DM Functional mobility and executive function in elderly diabetics and non-diabetics. Rev Bras Fisioter. 14, (2011), 491-197.

17. Pijpers E, Ferreira I, de Jongh RT, et al. Older individuals with diabetes have an increased risk of recurrent falls: analysis of potential mediating factors: the Longitudinal Ageing Study Amsterdam. Age Ageing. 41, (2011), 358-365.

18. Bruce D, Hunter M, Peters K, et al. Fear of falling is common in patients with type 2 diabetes and is associated with increased risk of falls. Age Ageing. 44, (2015), 687-690.

19. Chang JT, Ganz DA. Quality Indicators for Falls and Mobility Problems in Vulnerable Elders. J Am Geriatr Soc. 55, (2007), S327-S34. 
20. Harrell FE Jr, Lee KL, Mark DB. Multivariable prognostic models: issues in developing models, evaluating assumptions and adequacy, and measuring and reducing errors. Stat Med. 15, (1996), 361-387.

21. Mythili A, Kumar KD, Subrahmanyam KA, et al. A Comparative study of examination scores and quantitative sensory testing in diagnosis of diabetic polyneuropathy. I Int J Diabetes Dev Ctries. 30, (2010), 43-51.

22. Henderson EJ, Lord SR, Brodie MA, et al. Rivastigmine for gait stability in patients with Parkinson's disease (ReSPonD): a randomised, double-blind, placebo-controlled, phase 2 trial. Lancet Neurol. 15, (2016), 249-258.

23. Liu-Ambrose TY, Ashe MC, Graf P, et al. Increased risk of falling in older communitydwelling women with mild cognitive impairment. Phys Ther. 88, (2008), 1482-1491.

24. Vazzana R, Bandinelli S, Lauretani F, et al. Trail Making Test predicts physical impairment and mortality in older persons. J Am Geriatr Soc. 58, (2010), 719-723.

25. Lord SR, Menz HB, Tiedemann A. A physiological profile approach to falls risk assessment and prevention. Phys Ther. 83, (2003), 237-252.

26. Hazel CA, Elliott DB. The dependency of $\log$ MAR visual acuity measurements on chart design and scoring rule. Optom Vis Sci. 79, (2002), 788-792.

27. Tsai Y-J, Yang Y-C, Lu F-H, et al. Functional Balance and Its Determinants in Older People with Diabetes. PLoS One. (2016);11:e0159339-e.

28. Lord SR, Rogers MW, Howland A, Fitzpatrick R. Lateral stability, sensorimotor function and falls in older people. J Am Geriatr Soc. 47, (1999), 1077-1081.

29. Menant JC, Latt MD, Menz HB, et al. Postural sway approaches center of mass stability limits in Parkinson's disease. Movement disorders. Mov Disord. 26, (2011), 637-643.

30. Allet L, Kim H, Ashton-Miller J, et al. Frontal plane hip and ankle sensorimotor function, not age, predicts unipedal stance time. Muscle Nerve. 45, (2012), 578-585.

31. Frzovic D, Morris ME, Vowels L. Clinical tests of standing balance: performance of persons with multiple sclerosis. Arch Phys Med Rehabil. 81, (2000), 215-221.

32. Mariani B, Rochat S, Bula CJ, Aminian K. Heel and toe clearance estimation for gait analysis using wireless inertial sensors. IEEE Trans Biomed Eng.59, (2012), 3162-3270.

33. Hofheinz M, Mibs M. The Prognostic Validity of the Timed Up and Go Test With a Dual Task for Predicting the Risk of Falls in the Elderly. Gerontol Geriatr Med. (2016);2:2333721416637798.

34. Lamb SE, Jorstad-Stein EC, Hauer K, Becker C. Development of a common outcome data set for fall injury prevention trials: the Prevention of Falls Network Europe consensus. $J$ Am Geriatr Soc. 53, (2005), 1618-1622.

35. Yau RK, Strotmeyer ES, Resnick HE, et al. Diabetes and risk of hospitalized fall injury among older adults. Diabetes Care. 36, (2013), 3985-3991.

36. Azidah AK, Hasniza H, Zunaina E. Prevalence of Falls and Its Associated Factors among Elderly Diabetes in a Tertiary Center, Malaysia. Curr Gerontol Geriatr Res. (2012);539073.

37. Jørgensen V, Butler Forslund E, Opheim A, et al. Falls and fear of falling predict future falls and related injuries in ambulatory individuals with spinal cord injury: a longitudinal observational study. J Physiother. 63, (2017), 108-113.

38. Close JCT, Hooper R, Glucksman E, et al. Predictors of falls in a high risk population: results from the prevention of falls in the elderly trial (PROFET). Emerg Med J. 20, (2003), 421425. 
39. Ambrose AF, Paul G, Hausdorff JM. Risk factors for falls among older adults: A review of the literature. Maturitas. 75, (2013), 51-61.

40. Nelson JM, Dufraux K, Cook PF. The Relationship Between Glycemic Control and Falls in Older Adults. J Am Geriatr Soc. 55, (2007), 2041-2044.

41. Yang Y, Hu X, Zhang Q, Zou R. Diabetes mellitus and risk of falls in older adults: a systematic review and meta-analysis. Age Ageing. 45, (2016) 761-767.

42. Roman de Mettelinge T, Cambier D, Calders P, et al. Understanding the relationship between type 2 diabetes mellitus and falls in older adults: a prospective cohort study. PLoS One. (2013);8:e67055.

43. Alvarenga PP, Pereira DS, Anjos DMC. Mobilidade funcional e função executiva em idosos diabéticos e não diabéticos. Braz J Phys Ther. 14, (2010) 491-496.

44. Thanthrige R, Dassanayake S, Dissanayake D. Relationship between increased risk of falling and cognitive impairment in residents of an elderly home in the Colombo district. The Ceylon Med J. 59, (2014), 21-23.

45. Yang Y, Hu X, Zhang Q, Zou R. Diabetes mellitus and risk of falls in older adults: a systematic review and meta-analysis. Age Aging. 45, (2016), 761-767. 
Table 1

Demographic, medication, diabetes mellitus and fall-related measures for the non-faller, single faller and multiple faller groups (mean+SD unless stated)

\begin{tabular}{|c|c|c|c|c|}
\hline Variable & $\begin{array}{l}\text { No falls } \\
(n=66)\end{array}$ & $\begin{array}{l}\text { One fall } \\
(n=11)\end{array}$ & $\begin{array}{l}\text { Two plus } \\
\text { falls } \\
(n=26)\end{array}$ & IRR (95\% CI) \\
\hline \multicolumn{5}{|l|}{ Demographic } \\
\hline Age & $61.4 \underline{+5.6}$ & $64.0 \pm 5.5$ & $60.8 \pm 7.2$ & $0.99(0.94-1.05)$ \\
\hline Female, n (\%) & $40(\overline{60} .6)$ & $6(5 \overline{4} .5)$ & $22(8 \overline{4} .6)$ & $3.60(1.50-8.65)$ \\
\hline Height $(\mathrm{cm})$ & $157.6 \pm 8.0$ & $156.9 \pm 8.0$ & $\begin{array}{l}153.83 \pm 6 . \\
7\end{array}$ & $0.95(0.89-1.00)$ \\
\hline Weight, (kg) & $\begin{array}{l}63.09 \pm 9.2 \\
0\end{array}$ & $60.72 \pm 9.79$ & $\begin{array}{l}58.76 \pm 9.4 \\
9\end{array}$ & $0.96(0.92-1.002)$ \\
\hline BMI & $25.4(3.3)$ & $24.6(3.2)$ & $24.8(2.9)$ & $0.95(0.83-1.08)$ \\
\hline \multicolumn{5}{|l|}{ Medications } \\
\hline Number of medications & $6.7 \pm 2.6$ & $6.4 \pm 1.6$ & $7.4 \pm 2.9$ & $1.14(0.99-1.31)$ \\
\hline Insulin dependent, $\mathrm{n}(\%)$ & $18 \overline{(27.3)}$ & $1(\overline{9.1})$ & $12(46.1)$ & $2.23(1.00-5.00)$ \\
\hline \multicolumn{5}{|l|}{ Diabetes-related measures } \\
\hline HbA1c $(\%)$ & $8.1 \pm 1.5$ & $7.1 \pm 1.2$ & $8.9 \pm 2.3$ & $1.23(1.00-1.5)$ \\
\hline Symptom score & $1.2 \pm 1.3$ & $1.1 \pm 1.2$ & $2.0 \pm 1.4$ & $1.54(1.19-2.00)$ \\
\hline Examination score & $3.1 \pm 3.1$ & $1.7 \pm 2.2$ & $4.0 \pm 3.3$ & $1.13(1.00-1.27)$ \\
\hline Diagnosed neuropathy, n (\%) & $29(43.9)$ & $3(\overline{27} .3)$ & $19 \overline{(73.1)}$ & $2.52(1.17-5.41)$ \\
\hline \multicolumn{5}{|l|}{ Lower limb sensation } \\
\hline Tibial nerve velocity ${ }^{\#}\left(\mathrm{~ms}^{-1}\right)$ & $40.5 \pm 5.7$ & $40.7 \pm 3.7$ & $39.7 \pm 6.5$ & $0.99(0.92-1.06)$ \\
\hline VPT $(\mathrm{mV})$ & $27.6 \pm 14.1$ & $23.9 \pm 13.5$ & $30.9 \pm 15.7$ & $1.01(0.99-1.04)$ \\
\hline Tactile sensitivity ( $\lg 10 \mathrm{mg}$ force) & $4.13 \pm 0.76$ & $4.11 \pm 0.90$ & $4.12 \pm 0.93$ & $1.24(0.80-1.90)$ \\
\hline Proprioception (degrees) & $2.5 \pm 1.3$ & $2.3 \pm 0.9$ & $2.1+1.2$ & $0.77(0.56-1.07)$ \\
\hline \multicolumn{5}{|l|}{ Fall-related measures } \\
\hline $1+$ fall in previous year, $\mathrm{n}(\%)$ & $15(22.7)$ & $7(63.6)$ & $17(65.4)$ & $5.47(2.72-11.01)$ \\
\hline Fear of falling & $26.6 \pm 7.6$ & $25.4 \pm 7.7$ & $30.2 \pm 6.6$ & $1.07(1.01-1.13)$ \\
\hline
\end{tabular}


Table 2

Cognition, vision, strength, gait and mobility measures for the non-faller, single faller and multiple faller groups (mean+SD unless stated)

\begin{tabular}{|c|c|c|c|c|}
\hline Variable & $\begin{array}{l}\text { No falls } \\
(n=66)\end{array}$ & $\begin{array}{l}\text { One fall } \\
(n=11)\end{array}$ & $\begin{array}{l}\text { Two plus } \\
\text { falls } \\
(n=26)\end{array}$ & IRR (95\% CI) \\
\hline \multicolumn{5}{|l|}{ Cognition } \\
\hline MOCA (score) & $23.3 \pm 2.7$ & $22.6 \pm 3.5$ & $22.3 \pm 3.0$ & $0.90(0.78-1.04)$ \\
\hline $\begin{array}{l}\text { Simple reaction time } \\
(\mathrm{ms})\end{array}$ & $254 \pm 48$ & $257 \pm 45$ & $266+50$ & $1.003(0.99-1.01)$ \\
\hline Trails A (s) & $65.0 \pm 27.3$ & $65.8 \pm 8.7$ & $67.9 \pm 25.6$ & $1.00(0.98-1.01)$ \\
\hline Trails B (s) & $164 . \overline{7} \pm 79.5$ & $174 . \overline{6}+85.0$ & $190 . \overline{1} \pm 109.2$ & $1.00(0.99-1.00)$ \\
\hline Trails B-A (s) & $97.2 \pm 62.0$ & $97.3 \pm 54.4$ & $123.6 \pm 94.2$ & $1.00(0.99-1.00)$ \\
\hline \multicolumn{5}{|l|}{ Vision } \\
\hline Contrast sensitivity (dB) & $20.8 \pm 2.4$ & $19.4+1.9$ & $19.5 \pm 2.6$ & $0.82(0.69-0.97)$ \\
\hline $\begin{array}{l}\text { High contrast visual } \\
\text { acuity (MAR) }\end{array}$ & $1.66 \pm 1.00$ & $1.74 \pm 1.10$ & $1.59 \pm 0.68$ & $1.16(0.72-1.86)$ \\
\hline $\begin{array}{l}\text { Low contrast visual } \\
\text { acuity (MAR) }\end{array}$ & $4.46 \pm 3.66$ & $5.21 \pm 4.69$ & $4.54 \pm 3.48$ & $1.03(0.92-1.16)$ \\
\hline \multicolumn{5}{|l|}{ Strength } \\
\hline $\begin{array}{l}\text { Knee extension strength } \\
(\mathrm{kg})\end{array}$ & $23.7 \pm 8.0$ & $23.3 \pm 7.8$ & $20.1 \pm 5.6$ & $0.92(0.87-0.97)$ \\
\hline $\begin{array}{l}\text { Five time sit to stand } \\
\text { time (s) }\end{array}$ & $13.0 \pm 2.4$ & $13.8 \pm 2.5$ & $13.1 \pm 2.2$ & $1.10(0.93-1.31)$ \\
\hline \multicolumn{5}{|l|}{ Balance } \\
\hline Sway-EO-on floor $(\mathrm{cm})$ & $6.7 \pm 2.7$ & $7.2 \pm 1.9$ & $8.4 \pm 4.5$ & $1.09(0.99-1.21)$ \\
\hline Sway-EC-on foam $(\mathrm{cm})$ & $8.8 \pm 35$ & $9.7 \pm 55$ & $10 . \overline{8}+5.2$ & $1.10(1.02-1.19)$ \\
\hline Sway-EO-on floor $(\mathrm{cm})$ & $19 . \overline{5} \pm 91$ & $19.9 \pm 100$ & $21.8 \pm 9.7$ & $1.04(1.00-1.08)$ \\
\hline Sway-EC-on foam $(\mathrm{cm})$ & $45.5 \pm 18.3$ & $38.1 \pm 12.2$ & $48.5 \pm 18.7$ & $1.01(0.99-1.03)$ \\
\hline $\begin{array}{l}\text { Maximum balance range } \\
\text { (cm) }\end{array}$ & $13.5 \pm 2.3$ & $13.1 \pm 3.6$ & $13.3 \pm 2.6$ & $0.89(0.77-1.02)$ \\
\hline
\end{tabular}




\begin{tabular}{|c|c|c|c|c|}
\hline $\begin{array}{l}\text { Coordinated stability } \\
\text { (error score) }\end{array}$ & $10.4+8.7$ & $11.0+7.7$ & $12.9+8.7$ & $1.04(0.99-1.08)$ \\
\hline Unipedal stance time (s) & $21.7 \pm 10.1$ & $25.5 \pm 5.1$ & $18.9 \pm 10.0$ & $0.96(0.92-1.00)$ \\
\hline $\begin{array}{l}\text { Inability to maintain } \\
\text { tandem standing for } \\
\text { 30sec, } \mathrm{n}(\%)\end{array}$ & $10(\overline{15.2)}$ & $5(4 \overline{5.5)}$ & $8(30.8)$ & $3.35(1.47-7.72)$ \\
\hline $\begin{array}{l}\text { Gait and mobility } \\
\text { measures }\end{array}$ & & & & \\
\hline Gait velocity (m/s) & $1.40 \pm 0.24$ & $1.45 \pm 0.24$ & $1.30 \pm 0.18$ & $0.19(0.03-1.16)$ \\
\hline Stride length/height & $0.86 \pm 0.09$ & $0.88 \pm 0.09$ & $0.82 \pm 0.07$ & $\begin{array}{l}0.009(0.0001- \\
0.829)\end{array}$ \\
\hline Cadence (steps/min) & $121.3 \pm 10.2$ & $124.9 \pm 10.1$ & $120.4 \pm 8.2$ & $1.00(0.96-1.05)$ \\
\hline Gait variability $(\%)$ & $2.79 \pm 1.24$ & $2.43 \pm 1.01$ & $2.88 \pm 1.02$ & $1.21(0.83-1.76)$ \\
\hline TUG (s) & $7.6 \pm 1.9$ & $7.7 \pm 1.3$ & $8.2 \pm 1.9$ & $1.29(1.03-1.62)$ \\
\hline TUG cognitive (s) & $11.7 \pm 3.6$ & $11.5 \pm 2.8$ & $14.1 \pm 5.0$ & $1.13(1.02-1.24)$ \\
\hline
\end{tabular}

High scores in the MOCA, simple reaction time, trails, visual acuity, five time sit to stand time, sway, coordinated stability, gait variability and TUG tests and low scores in the contrast sensitivity, strength, maximal balance range, stride length, gait speed and cadence measures indicate poorer performances. Participants who were unable to perform a sway test due to poor balance were assigned mean $+3 \mathrm{SD}$ scores based on the available data for that variable.

\#n; no falls=59, one fall=11, two plus falls=21 (tibial nerve velocity was not detected in the remainder of participants) 
Figure 1- Proportion of falls in relation to number of falls risk factors

\section{Figure Legend}

Absolute risk of multiple falls in relation to number of risk factors in diabetes mellitus older adults. Risk factors comprised: presence of diagnosed neuropathy, vision contrast score $<20 \mathrm{~dB}$, knee extension strength score $<17.5 \mathrm{~kg}$ force, inability to maintain tandem balance for $30 \mathrm{~s}$, gait speed $<1.4 \mathrm{~m} / \mathrm{s}$. 